\title{
Concise synthesis of a buffalo milk pentasaccharide derivative
}

\author{
Guofeng Gu, ${ }^{a}$ Yuguo Du, a,* Jingqi Pan ${ }^{\mathrm{b}}$ \\ ${ }^{a}$ Research Center for Eco-Environmental Sciences, Academia Sinica, PO Box 2871, Beijing 100085, PR China \\ ${ }^{\mathrm{b}}$ College of Chemistry and Molecular Engineering, Peking University, Beijing 100871, PR China
}

Received 14 May 2002; accepted 5 June 2002

\begin{abstract}
An efficient synthesis of buffalo milk pentasaccharide derivative via a $3+2$ strategy is described. The use of a trisaccharide isopropyl thioglycoside as a latent glycosyl donor and the application of two well-defined regioselective glycosylations significantly simplified the target preparation. (C) 2002 Elsevier Science Ltd. All rights reserved.
\end{abstract}

Keywords: Glycosylation; Antigens; Thioglycosides; Oligosaccharides; Regioselectivity

Milk, which contains numerous complex carbohydrate components, is known as a good source of oligosaccharide antigens and bifidus factors for breastfed newborns. ${ }^{1}$ Monoclonal antibodies of several tumour cell lines or carbohydrate antigens have provided evidence that membrane glycoproteins or glycolipids, which may function as differentiation antigens or tumour-associate antigens, also occur as free oligosaccharides in human milk. ${ }^{2}$ Different types of milk since then have been analyzed for their oligosaccharides that have immunological properties. ${ }^{3}$ Recently, Deepak and coworkers ${ }^{4}$ isolated a novel pentasaccharide from immunostimulant oligosaccharide fraction of buffalo milk with the following structure: $\beta$-D-GlcNAc- $(1 \rightarrow 3)-\beta-D-$ Gal-( $1 \rightarrow 4)-\beta$-D-GlcNAc- $(1 \rightarrow 3)$-Gal-( $1 \rightarrow 4)$-D-Glc. $\quad$ A six-fold increase in haemagglutinating antibody (HA) titre and a two-fold increase in plaque-forming cell (PFC) count were reported in treated animals as compared with untreated controls. To investigate structure-activity relationships, we planned to prepare a monomer and a trimer of the above-mentioned pentasaccharide. We present here the concise chemical synthesis of this pentasaccharide derivative.

The efficient chemical synthesis of complex oligosaccharides requires highly convergent strategies in which

\footnotetext{
* Corresponding author. Tel.: + 86-10-62914475; fax: + 86-10-62923563

E-mail address: ygdu@mail.rcees.ac.cn (Y. Du).
}

well-designed glycosyl donors and acceptors are assembled involving a minimum of steps of reactions. Accordingly, the target molecule was retrosynthetically disconnected into a disaccharide acceptor $\mathbf{5}$ and a trisaccharide donor $\mathbf{1 4}$ as shown in Scheme 1. Thus, compound $\mathbf{1}^{5}$ was condensed with $\mathbf{3}^{6}$ in the presence of $N$-iodosuccinimide (NIS) and catalytic amount of trimethylsilyl triflate (TMSOTf) to afford disaccharide 4 in $89 \%$ yield. The stereochemical outcome is induced by the neighbouring-group participation of donor $\mathbf{1}$ and confirmed by the ${ }^{1} \mathrm{H}$ NMR spectroscopy of 4 (H-1: $\delta$ $4.49 \mathrm{ppm}, J_{1,2} 8.0 \mathrm{~Hz}$ ). Hydrolysis of 4 in aqueous $90 \%$ trifluoroacetic acid (TFA) furnished disaccharide acceptor 5 in good yield (84.5\%). Convergently, $\mathbf{1}$ was reacted with $p$-methoxyphenol (MPOH) with the promotion of NIS-TMSOTf in dry $\mathrm{CH}_{2} \mathrm{Cl}_{2}$ to give 2, which was treated with $90 \%$ TFA to generate diol $\mathbf{6}$ (63.7\% for two steps). Regioselective coupling of 6 with glucosamine imidate 7 in anhydrous $\mathrm{CH}_{2} \mathrm{Cl}_{2}$ in the presence of TMSOTf produced $\beta$ - $(1 \rightarrow 3)$-linked disaccharide $\mathbf{8}$ in $72 \%$ yield. Acetylation of $\mathbf{8}$ with acetic anhydride in pyridine gave $\mathbf{9}$ showing $\mathrm{H}-4$ at $\delta 5.63$ ppm $\left(J_{4,3} 3.4 \mathrm{~Hz}\right)$, which confirmed the correct regioselectivity. Ceric ammonium nitrate (CAN) promoted cleavage of anomeric MP of 9 was carried out smoothly in toluene- $\mathrm{MeCN}-\mathrm{H}_{2} \mathrm{O}$ co-solvent system $(\rightarrow \mathbf{1 0})$, followed by Schmidt activation using trichloroacetonitrile and DBU, to afford imidate $\mathbf{1 1}$ in $72.3 \%$ yield (based on 8 ). 
To save steps in protection-group manipulation on the anomeric centre, the isopropyl thioglycoside ${ }^{7}$ was designed as a latent glycosyl donor for the glucosamine residue. It was also reported ${ }^{8}$ that the $6-\mathrm{OH}$ benzoylated glucosamine unit decreased the activity of the group 4-OH while the ether-protected counterparts improved the yields greatly. As we expected, coupling of isopropyl thioglycoside $\mathbf{1 2}^{9}$ and $\mathbf{1 1}$ at $0{ }^{\circ} \mathrm{C}$ using TMSOTf as catalyst gave $\beta$ - $(1 \rightarrow 4)$-linked trisaccharide $\mathbf{1 3}$ $(71 \%)$, which was further acetylated with acetic anhydride in pyridine to furnish trisaccharide donor $\mathbf{1 4}$. Neither $\alpha$ nor $\beta$ product with $1 \rightarrow 3$ linkage $^{10}$ was detected in our experiments. 2D NMR experiments showed that the chemical shift of H-3 ${ }^{\mathrm{I}}$ moved downfield from $4.39 \mathrm{ppm}$ (in 13) to $5.64 \mathrm{ppm}$ (in 14), together with $\mathrm{H}-1^{\mathrm{II}}$ at $\delta 4.74 \mathrm{ppm}\left(J_{1,2} 8.1 \mathrm{~Hz}\right)$, confirming the formation of a $\beta-(1 \rightarrow 4)$ linkage. Glycosylation of isopropyl thioglycoside $\mathbf{1 4}$ and disaccharide diol $\mathbf{5}$ in the presence of NIS-TMSOTf at $-15^{\circ} \mathrm{C}$ completed the synthesis of pentasaccharide derivative $15(66 \%)$. ${ }^{1} \mathrm{H}-$ ${ }^{1} \mathrm{H}$ and ${ }^{1} \mathrm{H}-{ }^{13} \mathrm{C}$ COSY analyses assigned the peak at $\delta$ $81.25 \mathrm{ppm}\left({ }^{13} \mathrm{C}\right.$ NMR spectroscopy) as $\mathrm{C}-3^{\mathrm{II}}$. This downfield movement of C-3 $3^{\mathrm{II}}$, compared with signals of C-3 $3^{\mathrm{V}}(70.14 \mathrm{ppm}), \mathrm{C}-4^{\mathrm{II}}(67.67 \mathrm{ppm})$ and C-4 ${ }^{\mathrm{IV}}(69.29$ $\mathrm{ppm})$, indicated the $(1 \rightarrow 3)$-bond formation in the last glycosylation step. $\mathrm{H}-1^{\mathrm{III}}$ appeared at $\delta 5.31 \mathrm{ppm}$ in the ${ }^{1} \mathrm{H}$ NMR spectrum $\left(J_{1,2} 8.4 \mathrm{~Hz}\right)$, which confirmed the $\beta$ stereoselectivity.

In conclusion, we have described the concise synthesis of the methyl glycoside derivative corresponding to

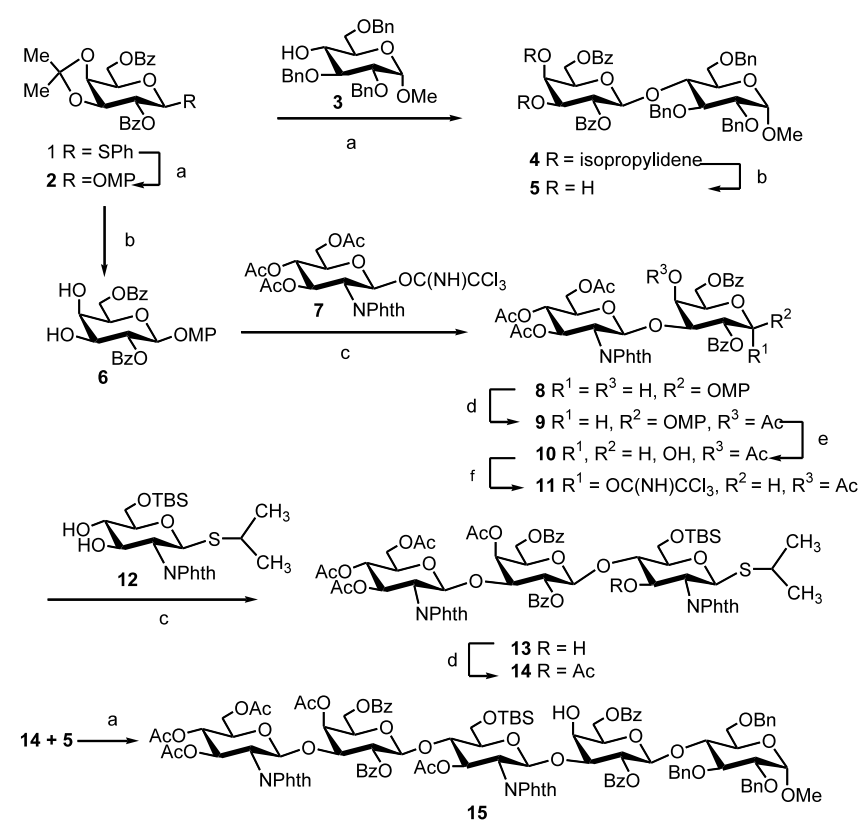

Scheme 1. Reagents and conditions: (a) NIS, TMSOTf; $89.1 \%$ for 4; $65.5 \%$ for 15; (b) $90 \%$ TFA; $84.5 \%$ for 5 ; $63.7 \%$ for 6 (from 1); (c) TMSOTf, $\mathrm{CH}_{2} \mathrm{Cl}_{2}, 71.9 \%$ for $8 ; 71.2 \%$ for 13 ; (d) $\mathrm{Ac}_{2} \mathrm{O}$, Pyr; (e) CAN, 3:4:3 Toluene-MeCN-water; (f) $\mathrm{Cl}_{3} \mathrm{CCN}, \mathrm{DBU}, 78.7 \%$ from 9. the bioactive buffalo milk pentasaccharide. Two welldefined regioselective glycosylation steps and the use of isopropyl thioglycoside as the latent donor simplified the protecting-group manipulations in the synthesis and the strategic principle described here is currently employed in the exploring of trimeric analogue preparation.

\section{Experimental}

General methods.-Optical rotations were determined at $20^{\circ} \mathrm{C}$ with a Perkin-Elmer Model 241-Mc automatic polarimeter. Melting points were determined with a "Mel-Temp" apparatus. ${ }^{1} \mathrm{H}$ NMR, ${ }^{13} \mathrm{C}$ NMR and ${ }^{1} \mathrm{H}-{ }^{1} \mathrm{H},{ }^{1} \mathrm{H}-{ }^{13} \mathrm{C}$ COSY spectra were recorded with ARX 400 spectrometers for solutions in $\mathrm{CDCl}_{3}$. Chemical shifts are given in ppm downfield from internal $\mathrm{Me}_{4} \mathrm{Si}$. Mass spectra were measured using MALDITOF-MS with $\alpha$-cyano-4-hydroxycinnamic acid (CCA) as matrix. Thin-layer chromatography (TLC) was performed on silica gel $\mathrm{HF}_{254}$ with detection by charring with $30 \%(\mathrm{v} / \mathrm{v}) \mathrm{H}_{2} \mathrm{SO}_{4}$ in $\mathrm{MeOH}$, or in some cases by a UV detector.

Methyl 2,6-di-O-benzoyl-3,4-di-O-isopropylidene- $\beta$ D - galactopyranosyl - $(1 \rightarrow 4)$ - 2,3,4 - tri - O - benzyl - $\alpha$ - Dglucopyranoside (4). - To a solution of $\mathbf{1}(290 \mathrm{mg}, 0.558$ $\mathrm{mmol})$ and 3 (246 mg, $0.526 \mathrm{mmol})$ in anhyd $\mathrm{CH}_{2} \mathrm{Cl}_{2}(4$ $\mathrm{mL})$ was added NIS (310 $\mathrm{mg}, 1.38 \mathrm{mmol})$ and $\mathrm{Me}_{3} \mathrm{SiOTf}(20 \mu \mathrm{L}, 0.11 \mathrm{mmol})$ under an $\mathrm{N}_{2}$ atmosphere at $0{ }^{\circ} \mathrm{C}$. The mixture was stirred under these conditions for $1 \mathrm{~h}$, then at $\mathrm{rt}$ for another $30 \mathrm{~min}$, at the end of which time TLC (6:1 petroleum ether-EtOAc) indicated that starting material $\mathbf{1}$ was completely consumed. The reaction mixture was neutralized with $\mathrm{Et}_{3} \mathrm{~N}$ and concentrated. Column chromatography (5:1 petroleum ether-EtOAc) of the residue gave $4(410 \mathrm{mg}$, $89.1 \%)$ as a syrup: $[\alpha]_{\mathrm{D}}+18^{\circ}\left(c 0.75, \mathrm{CHCl}_{3}\right) ;{ }^{1} \mathrm{H}$ NMR: $1.34,1.60\left(2 \mathrm{~s}, 2 \times 3 \mathrm{H}, 2 \mathrm{CH}_{3}\right), 3.27(\mathrm{~s}, 3 \mathrm{H}$, $\mathrm{OCH}_{3}$ ), 3.35-3.52 (m, $\left.3 \mathrm{H}, \mathrm{H}-6 \mathrm{a}, \mathrm{H}-6 \mathrm{~b}, \mathrm{H}-5\right), 3.67$ (dd, $\left.1 \mathrm{H}, J_{2,1} 2.5, J_{2,3} 9.2 \mathrm{~Hz}, \mathrm{H}-2\right), 3.80-3.92(\mathrm{~m}, 3 \mathrm{H}, \mathrm{H}-3$, H-4, H-5'), 4.04 (dd, $1 \mathrm{H}, J_{3^{\prime}, 4^{\prime}} 4.7, J_{3^{\prime}, 2^{\prime}} 7.5 \mathrm{~Hz}, \mathrm{H}-3^{\prime}$ ), $4.16\left(\mathrm{~d}, 1 \mathrm{H}, \mathrm{H}-4^{\prime}\right), 4.29(\mathrm{~d}, 1 \mathrm{H}, J 12.2 \mathrm{~Hz}$, one proton of $\left.\mathrm{PhCH}_{2}\right), 4.36\left(\mathrm{dd}, 1 \mathrm{H}, J_{6 \mathrm{a}^{\prime}, 6 \mathrm{~b}^{\prime}} 11.3, J_{6 \mathrm{a}^{\prime}, 5^{\prime}} 7.0 \mathrm{~Hz}\right.$, H-6a'), 4.49 (d, $\left.1 \mathrm{H}, J_{1^{\prime}, 2^{\prime}} 8.0 \mathrm{~Hz}, \mathrm{H}-1^{\prime}\right), 4.52-4.58(\mathrm{~m}$, $2 \mathrm{H}, \mathrm{H}-6 \mathrm{~b}, \mathrm{H}-1), 4.60,4.68,4.77,4.78,5.03(5 \mathrm{~d}, 5 \mathrm{H}$, $\left.J 12.2,10.7 \mathrm{~Hz}, \mathrm{PhCH}_{2}\right), 5.21\left(\mathrm{t}, 1 \mathrm{H}, J_{2^{\prime}, 3^{\prime}} 7.5, J_{2^{\prime}, 1^{\prime}} 8.0\right.$ $\left.\mathrm{Hz}, \mathrm{H}-2^{\prime}\right), 7.25-8.04$ (m, $\left.30 \mathrm{H}, \mathrm{Ph}\right)$. MALDITOF-MS Calcd for $\mathrm{C}_{51} \mathrm{H}_{54} \mathrm{O}_{13}: 874[\mathrm{M}]$; Found: $897.3[\mathrm{M}+\mathrm{Na}]^{+}$. Methyl 2,6-di-O-benzoyl- $\beta$-D-galactopyranosyl- $(1 \rightarrow$ 4)-2,3,4-tri-O-benzyl- $\alpha$-D-glucopyranoside (5). - A solution of compound 4 (400 $\mathrm{mg}, 0.458 \mathrm{mmol}$ ) in $90 \%$ TFA $(5 \mathrm{~mL})$ was stirred at $\mathrm{rt}$ for $15 \mathrm{~min}$, then neutralized with aq $\mathrm{NaHCO}_{3}$ and extracted with $\mathrm{CH}_{2} \mathrm{Cl}_{2}(2 \times 20$ $\mathrm{mL}$ ). The organic phases were combined and concentrated. The residue was purified on a silica gel column 
using 2:1 petroleum ether-EtOAc as eluent to give foamy 5 (323 mg, 84.5\%): $[\alpha]_{\mathrm{D}}+6^{\circ}\left(c 2.4, \mathrm{CHCl}_{3}\right) ;{ }^{1} \mathrm{H}$ NMR: 3.29 (s, $\left.3 \mathrm{H}, \mathrm{OCH}_{3}\right), 3.45-3.58(\mathrm{~m}, 5 \mathrm{H}, \mathrm{H}-6 \mathrm{a}$, H-6b, H-5, H-3', H-5'), 3.70 (dd, $1 \mathrm{H}, J_{2,1} 3.4, J_{2,3} 10.8$ $\mathrm{Hz}, \mathrm{H}-2$ ), 3.80-3.96 (m, 3 H, H-3, H-4, H-4'), 4.17 (dd, $\left.1 \mathrm{H}, J_{6 \mathrm{a}^{\prime}, 6 \mathrm{~b}^{\prime}} 11.2, J_{6 \mathrm{a}^{\prime}, 5^{\prime}} 5.7 \mathrm{~Hz}, \mathrm{H}-6 \mathrm{a}^{\prime}\right), 4.31(\mathrm{~d}, 1 \mathrm{H}, J$ $12.8 \mathrm{~Hz}$, one proton of $\left.\mathrm{PhCH}_{2}\right), 4.52\left(\mathrm{dd}, 1 \mathrm{H}, J_{6 \mathrm{~b}^{\prime}, 5^{\prime}} 7.8\right.$ $\left.\mathrm{Hz}, \mathrm{H}-6 \mathrm{~b}^{\prime}\right), 4.56$ (d, $\left.1 \mathrm{H}, J_{1,2} 3.4 \mathrm{~Hz}, \mathrm{H}-1\right), 4.59$ (d, $1 \mathrm{H}$, $\left.J_{1^{\prime}, 2^{\prime}} 8.2 \mathrm{~Hz}, \mathrm{H}-1^{\prime}\right), 4.63,4.76,4.78,4.84,4.98(5 \mathrm{~d}, 5 \mathrm{H}$, $J$ 12.3, 12.8, $\left.10.7 \mathrm{~Hz}, \mathrm{PhCH}_{2}\right), 5.17\left(\mathrm{t}, 1 \mathrm{H}, J_{2^{\prime}, 3^{\prime}} 9.2\right.$, $\left.J_{2^{\prime}, 1^{\prime}} 8.2 \mathrm{~Hz}, \mathrm{H}-2^{\prime}\right), 7.25-8.04(\mathrm{~m}, 30 \mathrm{H}, \mathrm{Ph}) ;{ }^{13} \mathrm{C} \mathrm{NMR}$ $\left(100 \mathrm{MHz}, \mathrm{CDCl}_{3}\right): 55.31\left(\mathrm{OCH}_{3}\right), 62.03\left(\mathrm{C}-6^{\prime}\right), 67.90$ (C-6), 68.34 (C-4'), 69.56 (C-3'), 71.97 (C-2'), 72.68 $\left(\mathrm{C}-5^{\prime}\right), 73.49\left(\mathrm{PhCH}_{2}\right), 73.50\left(\mathrm{PhCH}_{2}\right), 74.27(\mathrm{C}-5)$, $75.28\left(\mathrm{PhCH}_{2}\right), 76.48(\mathrm{C}-3), 79.02$ (C-2), 79.98 (C-4), 98.34 (C-1), 100.10 (C-1'), 166.50, 166.65 (2 C, PhCO); MALDITOF-MS Calcd for $\mathrm{C}_{48} \mathrm{H}_{50} \mathrm{O}_{13}: 834$ [M]; Found $857.4[\mathrm{M}+\mathrm{Na}]^{+}, 873.4[\mathrm{M}+\mathrm{K}]^{+}$. Anal. Calcd for $\mathrm{C}_{48} \mathrm{H}_{50} \mathrm{O}_{13}$ : C, 69.06; H, 6.04. Found: C, 68.84; H, 6.13. $\mathrm{p}$-Methoxyphenyl 2,6-di-O-benzoyl- $\beta$-D-galactopyranoside (6). - To a solution of $1(1.46 \mathrm{~g}, 2.81 \mathrm{mmol})$ and $p$-methoxyphenol $(418 \mathrm{mg}, 3.37 \mathrm{mmol})$ in anhyd $\mathrm{CH}_{2} \mathrm{Cl}_{2}(10 \mathrm{~mL})$ was added NIS $(948 \mathrm{mg}, 4.22 \mathrm{mmol})$ and $\mathrm{Me}_{3} \mathrm{SiOTf}(31 \mu \mathrm{L}, 0.17 \mathrm{mmol})$ under $\mathrm{N}_{2}$ atmosphere at $-15^{\circ} \mathrm{C}$. The mixture was stirred under these conditions for $1 \mathrm{~h}$, at the end of which time TLC (5:1 petroleum ether-EtOAc) indicated that starting material 1 was completely consumed. The reaction mixture was neutralized with $\mathrm{Et}_{3} \mathrm{~N}$ and concentrated. The above residue was dissolved into $90 \%$ TFA $(10 \mathrm{~mL})$, stirred at rt for $20 \mathrm{~min}$, then co-evaporated with toluene under diminished pressure to dryness. Purification of the residue on a silica gel column using 1:1 petroleum ether-EtOAc as eluent gave $6(884 \mathrm{mg}, 63.7 \%)$ as a white solid: $[\alpha]_{\mathrm{D}}-9^{\circ}\left(c 0.9, \mathrm{CHCl}_{3}\right)$; ${ }^{1} \mathrm{H}$ NMR: 3.71 (s, $3 \mathrm{H}, \mathrm{OCH}_{3}$ ), 3.94 (dd, $1 \mathrm{H}, J_{2,3} 9.5, J_{3,4} 3.5 \mathrm{~Hz}, \mathrm{H}-3$ ), $4.01\left(\mathrm{dd}, 1 \mathrm{H}, J_{5,6 \mathrm{a}} 7.6, J_{5,6 \mathrm{~b}} 5.4 \mathrm{~Hz}, \mathrm{H}-5\right), 4.11(\mathrm{~d}, 1 \mathrm{H}$, $\mathrm{H}-4), 4.65$ (dd, $\left.1 \mathrm{H}, J_{6 \mathrm{a}, 6 \mathrm{~b}} 11.5 \mathrm{~Hz}, \mathrm{H}-6 \mathrm{a}\right), 4.75$ (dd, $1 \mathrm{H}$, H-6b), 5.03 (d, $\left.1 \mathrm{H}, J_{1,2} 8.0 \mathrm{~Hz}, \mathrm{H}-1\right), 5.44(\mathrm{dd}, 1 \mathrm{H}$, $\mathrm{H}-2), 6.65(\mathrm{~d}, 2 \mathrm{H}, \mathrm{Ph}), 6.95$ (d, $2 \mathrm{H}, \mathrm{Ph}), 7.40-8.08$ (m, $10 \mathrm{H}, \mathrm{Ph})$

p-Methoxyphenyl 3,4,6-tri-O-acetyl-2-deoxy-2-phthalimido- $\beta$-D-glucopyranosyl- $(1 \rightarrow 3)$-2,6-di-O-benzo$y l$ - $\beta$-D-galactopyranoside (8). - Compound $6(900 \mathrm{mg}$, $1.82 \mathrm{mmol})$ and $7(1.10 \mathrm{~g}, 1.90 \mathrm{mmol})$ were pre-dried in one flask under vacuum at $60{ }^{\circ} \mathrm{C}$ for $2 \mathrm{~h}$. The mixture was then dissolved in $\mathrm{CH}_{2} \mathrm{Cl}_{2}(10 \mathrm{~mL})$. To the solution was added $\mathrm{Me}_{3} \operatorname{SiOTf}(36 \mu \mathrm{L}, 0.20 \mathrm{mmol})$ at $\mathrm{rt}$ under an $\mathrm{N}_{2}$ atmosphere. The mixture was stirred for $1.5 \mathrm{~h}$, then neutralized with $\mathrm{Et}_{3} \mathrm{~N}$, concentrated under reduced pressure, and purified on a silica gel column with 1:1 petroleum ether-EtOAc as the eluents to give 8 (954 $\mathrm{mg}, 71.9 \%$, based on recovered $\mathbf{5}$ ) as crystals: $\mathrm{mp}$ $203-205^{\circ} \mathrm{C} ;[\alpha]_{\mathrm{D}}+50^{\circ}\left(c \quad 0.75, \mathrm{CHCl}_{3}\right) ;{ }^{1} \mathrm{H}$ NMR: $1.79,2.05,2.13\left(3 \mathrm{~s}, 3 \times 3 \mathrm{H}, 3 \mathrm{CH}_{3} \mathrm{CO}\right), 3.65(\mathrm{~s}, 3 \mathrm{H}$, $\mathrm{OCH}_{3}$ ), 3.85-4.08 (m, 3 H, H-3, H-5, H-5'), 4.16-4.34 $\left(\mathrm{m}, 3 \mathrm{H}, \mathrm{H}-4, \mathrm{H}-6 \mathrm{a}^{\prime}, \mathrm{H}-6 \mathrm{~b}^{\prime}\right), 4.38$ (t, $1 \mathrm{H}, J_{2^{\prime}, 3^{\prime}} 10.7, J_{2^{\prime}, 1^{\prime}}$ $\left.8.2 \mathrm{~Hz}, \mathrm{H}-2^{\prime}\right), 4.68\left(\mathrm{dd}, 1 \mathrm{H}, J_{6 \mathrm{a}, 6 \mathrm{~b}} 11.4, J_{6 \mathrm{a}, 5} 7.9 \mathrm{~Hz}\right.$, H-6a), 4.73 (dd, $\left.1 \mathrm{H}, J_{6 \mathrm{~b}, 5} 4.3 \mathrm{~Hz}, \mathrm{H}-6 \mathrm{~b}\right), 4.83$ (d, $1 \mathrm{H}$, $\left.J_{1,2} 8.0 \mathrm{~Hz}, \mathrm{H}-1\right), 5.14\left(\mathrm{t}, 1 \mathrm{H}, J_{4^{\prime}, 3^{\prime}}=J_{4^{\prime}, 5^{\prime}}=9.5 \mathrm{~Hz}\right.$, H-4'), 5.54-5.72 (m, 3 H, H-2, H-1', H-3'), 6.53 (d, 2 H, $\mathrm{Ph}), 6.76(\mathrm{~d}, 2 \mathrm{H}, \mathrm{Ph}), 7.17-8.09(\mathrm{~m}, 14 \mathrm{H}, \mathrm{Ph})$. Anal. Calcd for $\mathrm{C}_{47} \mathrm{H}_{45} \mathrm{NO}_{18}$ : C, 69.91; H, 4.97. Found: C, 70.12; H, 5.09.

p-Methoxyphenyl 3,4,6-tri-O-acetyl-2-deoxy-2-phthalimido- $\beta$-D-glucopyranosyl- $(1 \rightarrow 3)$ - 4 -O-acetyl-2,6di-O-benzoyl- $\beta$-D-galactopyranoside (9). - To a solution of compound $8(915 \mathrm{mg}, 1.00 \mathrm{mmol})$ in pyridine (4 $\mathrm{mL})$ was added $\mathrm{Ac}_{2} \mathrm{O}(2 \mathrm{~mL})$. The mixture was stirred at $\mathrm{rt}$ for about $12 \mathrm{~h}$, then co-evaporated with toluene under diminished pressure to remove pyridine. Column chromatography (2:1 petroleum ether-EtOAc) of the residue gave crystalline 9 (879 $\mathrm{mg}, 91.8 \%): \mathrm{mp} \mathrm{210-}$ $212^{\circ} \mathrm{C} ;[\alpha]_{\mathrm{D}}+53^{\circ}\left(\mathrm{c} 1.4, \mathrm{CHCl}_{3}\right) ;{ }^{1} \mathrm{H}$ NMR: 1.78, 2.03, $2.12,2.23\left(4 \mathrm{~s}, 4 \times 3 \mathrm{H}, 4 \mathrm{CH}_{3} \mathrm{CO}\right), 3.64(\mathrm{~s}, 3 \mathrm{H}$, $\left.\mathrm{OCH}_{3}\right), 3.80-3.84\left(\mathrm{~m}, 1 \mathrm{H}, \mathrm{H}-5^{\prime}\right), 4.03-4.14(\mathrm{~m}, 2 \mathrm{H}$, $\mathrm{H}-3, \mathrm{H}-5), 4.18\left(\mathrm{dd}, 1 \mathrm{H}, J_{6 \mathrm{a}^{\prime}, 6 \mathrm{~b}^{\prime}} 11.9, J_{6 \mathrm{a}^{\prime}, 5^{\prime}} 4.2 \mathrm{~Hz}\right.$, H-6a'), 4.25 (dd, $\left.1 \mathrm{H}, J_{2^{\prime}, 3^{\prime}} 10.7, J_{2^{\prime}, 1^{\prime}} 8.2 \mathrm{~Hz}, \mathrm{H}-2^{\prime}\right), 4.33$ $\left(\mathrm{dd}, 1 \mathrm{H}, J_{6 \mathrm{~b}^{\prime}, 5^{\prime}} 5.7 \mathrm{~Hz}, \mathrm{H}-6 \mathrm{~b}^{\prime}\right), 4.41\left(\mathrm{dd}, 1 \mathrm{H}, J_{6 \mathrm{a}, 6 \mathrm{~b}} 11.4\right.$, $\left.J_{6 \mathrm{a}, 5} 7.9 \mathrm{~Hz}, \mathrm{H}-6 \mathrm{a}\right), 4.52$ (dd, $\left.1 \mathrm{H}, J_{6 \mathrm{~b}, 5} 4.3 \mathrm{~Hz}, \mathrm{H}-6 \mathrm{~b}\right)$, $4.87\left(\mathrm{~d}, 1 \mathrm{H}, J_{1,2} 8.0 \mathrm{~Hz}, \mathrm{H}-1\right), 5.16\left(\mathrm{t}, 1 \mathrm{H}, J_{4^{\prime}, 3^{\prime}}=\right.$ $\left.J_{4^{\prime}, 5^{\prime}}=9.3 \mathrm{~Hz}, \mathrm{H}-4^{\prime}\right), 5.48-5.57\left(\mathrm{~m}, 2 \mathrm{H}, \mathrm{H}-2, \mathrm{H}-1^{\prime}\right)$, $5.63\left(\mathrm{~d}, 1 \mathrm{H}, J_{4,3} 3.4 \mathrm{~Hz}, \mathrm{H}-4\right), 5.67\left(\mathrm{t}, 1 \mathrm{H}, J_{3^{\prime}, 4^{\prime}} 9.3\right.$, $\left.J_{3^{\prime}, 2^{\prime}} 10.7 \mathrm{~Hz}, \mathrm{H}-3^{\prime}\right), 6.50(\mathrm{~d}, 2 \mathrm{H}, \mathrm{Ph}), 6.74(\mathrm{~d}, 2 \mathrm{H}, \mathrm{Ph})$, 7.26-8.08 (m, $14 \mathrm{H}, \mathrm{Ph})$. Anal. Calcd for $\mathrm{C}_{49} \mathrm{H}_{47} \mathrm{NO}_{19}$ : C, 61.70; H, 4.97. Found: C, 61.64; H, 5.13.

3,4,6-Tri-O-acetyl-2-deoxy-2-phthalimido- $\beta$-D-glucopyranosyl- $(1 \rightarrow 3)-4-\mathrm{O}$-acetyl-2,6-di-O-benzoyl- $\beta$-Dgalactopyranosyl trichloroacetimidate (11). - Ceric ammonium nitrate (CAN; $1.22 \mathrm{~g}, 2.23 \mathrm{mmol}$ ) was added to a solution of $9(850 \mathrm{mg}, 0.892 \mathrm{mmol})$ in $3: 4: 3$ toluene- $\mathrm{MeCN}-\mathrm{H}_{2} \mathrm{O}(30 \mathrm{~mL})$, and the mixture was stirred at $\mathrm{rt}$ for $2 \mathrm{~h}$. An additional amount of CAN (734 mg, $1.34 \mathrm{mmol}$ ) was added, and the stirring was continued for another $2 \mathrm{~h}$, at the end of which time TLC (1:1 petroleum-EtOAc) indicated the completion of the reaction. The resulting mixture was diluted with EtOAc, washed successively with water, satd aq $\mathrm{NaHCO}_{3}$, and brine. The organic phase was dried over anhydrous $\mathrm{Na}_{2} \mathrm{SO}_{4}$ and concentrated, then subjected to silica gel column chromatography $(1: 1$ petroleum ether-EtOAc). The product generated above was dissolved in anhydrous $\mathrm{CH}_{2} \mathrm{Cl}_{2}(5 \mathrm{~mL})$. To the solution was added trichloroacetonitrile $(0.45 \mathrm{~mL}, 4.46 \mathrm{mmol})$ and DBU $(45 \mu \mathrm{L}, 0.45 \mathrm{mmol})$ at $\mathrm{rt}$. The mixture was stirred for $2 \mathrm{~h}$, then concentrated, and the residue was subjected to a silica gel column (1.5:1 petroleum etherEtOAc) to afford syrupy $11(696 \mathrm{mg}, 78.7 \%$ for two steps): $[\alpha]_{\mathrm{D}}+96^{\circ}\left(c 1.5, \mathrm{CHCl}_{3}\right) ;{ }^{1} \mathrm{H}$ NMR: $1.80,2.02$, $2.12,2.20\left(4 \mathrm{~s}, 4 \times 3 \mathrm{H}, 4 \mathrm{CH}_{3} \mathrm{CO}\right), 3.85-3.92(\mathrm{~m}, 1 \mathrm{H}$, H-5'), 4.15-4.25 (m, 2 H, H-3, H-2'), 4.32-4.44 (m, 4 H, H-5, H-6a, H-6a', H-6b'), 4.55 (dd, 1 H, J $J_{6 \mathrm{~b}, 6 \mathrm{a}} 12.3$, 
$\left.J_{6 \mathrm{~b}, 5} 5.0 \mathrm{~Hz}, \mathrm{H}-6 \mathrm{~b}\right), 5.17\left(\mathrm{t}, 1 \mathrm{H}, J_{4^{\prime}, 3^{\prime}} 9.2, J_{4^{\prime}, 5^{\prime}} 9.5 \mathrm{~Hz}\right.$, $\left.\mathrm{H}-4^{\prime}\right), 5.46\left(\mathrm{dd}, 1 \mathrm{H}, J_{2,3} 10.5, J_{2,1} 3.8 \mathrm{~Hz}, \mathrm{H}-2\right), 5.52(\mathrm{~d}$, $\left.1 \mathrm{H}, J_{1^{\prime}, 2^{\prime}} 3.8 \mathrm{~Hz}, \mathrm{H}-1^{\prime}\right), 5.70-5.77\left(\mathrm{~m}, 2 \mathrm{H}, \mathrm{H}-4, \mathrm{H}-3^{\prime}\right)$, $6.52\left(\mathrm{~d}, 1 \mathrm{H}, J_{1,2} 3.8 \mathrm{~Hz}, \mathrm{H}-1\right), 7.28-8.01(\mathrm{~m}, 14 \mathrm{H}, \mathrm{Ph})$, 8.34 (s, $1 \mathrm{H}, \mathrm{NH})$. Anal. Calcd for $\mathrm{C}_{44} \mathrm{H}_{41} \mathrm{Cl}_{3} \mathrm{~N}_{2} \mathrm{O}_{18}$ : C, 53.25; H, 4.17. Found: C, 53.08; H, 4.22.

Isopropyl 3,4,6-tri-O-acetyl-2-deoxy-2-phthalimido$\beta$-D-glucopyranosyl- $(1 \rightarrow 3)-4-\mathrm{O}$-acetyl-2,6-di-O-benzo$y l$ - $\beta$-D-galactopyranosyl-( $1 \rightarrow 4)$ - 6 -O-tert-butyldimethylsilyl-2-deoxy-2-phthalimido-1-thio- $\beta$-D-glucopyranoside (13). - To a solution of compound $11(660 \mathrm{mg}$, $0.666 \mathrm{mmol})$ and $12(300 \mathrm{mg}, 0.623 \mathrm{mmol})$ in anhyd $\mathrm{CH}_{2} \mathrm{Cl}_{2}(5 \mathrm{~mL})$ was added $\mathrm{Me}_{3}$ SiOTf $(12 \mu \mathrm{L}, 0.067$ mmol) under an $\mathrm{N}_{2}$ atmosphere at $0{ }^{\circ} \mathrm{C}$. The mixture was stirred under these conditions for $1 \mathrm{~h}$, neutralized with $\mathrm{Et}_{3} \mathrm{~N}$ and concentrated under reduced pressure. The residue was purified on a silica gel column with 1.3:1:0.1 petroleum ether-EtOAc-toluene as the eluent to give $13\left(582 \mathrm{mg}, 71.2^{\%}\right)$ as a syrup: $[\alpha]_{\mathrm{D}}+47^{\circ}(c 1$, $\left.\mathrm{CHCl}_{3}\right)$; ${ }^{1} \mathrm{H}$ NMR: $-0.17,-0.16(2 \mathrm{~s}, 2 \times 3 \mathrm{H}, 2$ $\left.\mathrm{SiCH}_{3}\right), 0.73\left(\mathrm{~s}, 9 \mathrm{H}, \mathrm{C}\left(\mathrm{CH}_{3}\right)_{3}\right), 1.10,1.12(2 \mathrm{~d}, 2 \times 3 \mathrm{H}$, $J$ 6.6, $\left.6.9 \mathrm{~Hz}, \mathrm{CH}\left(\mathrm{CH}_{3}\right)_{2}\right), 1.76,2.00,2.13,2.18(4 \mathrm{~s}$, $\left.4 \times 3 \mathrm{H}, 4 \mathrm{CH}_{3} \mathrm{CO}\right), 3.00\left(\mathrm{~m}, 1 \mathrm{H}, \mathrm{CH}\left(\mathrm{CH}_{3}\right)_{2}\right), 3.24-$ $3.42\left(\mathrm{~m}, 3 \mathrm{H}, J_{6 \mathrm{a}, 6 \mathrm{~b}} 10.4 \mathrm{~Hz}, \mathrm{H}-6 \mathrm{a}^{\mathrm{I}}, \mathrm{H}-6 \mathrm{~b}^{\mathrm{I}}, \mathrm{H}-5^{\mathrm{I}}\right), 3.57$ (t, $\left.1 \mathrm{H}, J_{4,3}=J_{4,5}=8.7 \mathrm{~Hz}, \mathrm{H}-4^{\mathrm{I}}\right), 3.82\left(\mathrm{~m}, 1 \mathrm{H}, \mathrm{H}-5^{\mathrm{III}}\right)$, $3.88-4.25$ (m, $6 \mathrm{H}, \mathrm{H}-5^{\mathrm{II}}, \mathrm{H}-6 \mathrm{a}^{\mathrm{III}}, \mathrm{H}-6 \mathrm{~b}^{\mathrm{III}}, \mathrm{H}-2^{\mathrm{I}}, \mathrm{H}-2^{\mathrm{III}}$, $\left.\mathrm{H}-3^{\mathrm{II}}\right), 4.34$ (br d, $\left.1 \mathrm{H}, J_{6 \mathrm{a}, 6 \mathrm{~b}} 11.6 \mathrm{~Hz}, \mathrm{H}-6 \mathrm{a}^{\mathrm{II}}\right), 4.39$ (t, 1 $\left.\mathrm{H}, J_{3,4} 8.7, J_{3,2} 10.1 \mathrm{~Hz}, \mathrm{H}-3^{\mathrm{I}}\right), 4.61-4.72(\mathrm{~m}, 2 \mathrm{H}$, $\left.\mathrm{H}-1^{\mathrm{II}}, \mathrm{H}-6 \mathrm{~b}^{\mathrm{II}}\right), 5.14\left(\mathrm{t}, 1 \mathrm{H}, J_{4,3}=J_{4,5}=9.3 \mathrm{~Hz}, \mathrm{H}-4^{\mathrm{III}}\right)$, $5.26\left(\mathrm{~d}, 1 \mathrm{H}, J_{1,2} 10.6 \mathrm{~Hz}, \mathrm{H}-1^{\mathrm{I}}\right), 5.34($ br t, $1 \mathrm{H}$, $\left.J_{2,3}=J_{2,1}=8.7 \mathrm{~Hz}, \mathrm{H}-2^{\mathrm{II}}\right), 5.47\left(\mathrm{~d}, 1 \mathrm{H}, J_{1,2} 8.2 \mathrm{~Hz}\right.$, $\mathrm{H}-1^{\mathrm{III}}$ ), 5.57 (br s, $1 \mathrm{H}, \mathrm{H}-4^{\mathrm{II}}$ ), 5.64 (t, $1 \mathrm{H}, J_{3,2} 10.6, J_{3,4}$ $\left.9.3 \mathrm{~Hz}, \mathrm{H}-3^{\mathrm{III}}\right), 7.26-8.05(\mathrm{~m}, 18 \mathrm{H}, \mathrm{Ph})$. Anal. Calcd for $\mathrm{C}_{65} \mathrm{H}_{74} \mathrm{~N}_{2} \mathrm{O}_{23} \mathrm{SSi}$ : C, 59.54; $\mathrm{H}$, 5.69. Found: $\mathrm{C}$, $59.21 ; \mathrm{H}, 5.50$.

Isopropyl 3,4,6-tri-O-acetyl-2-deoxy-2-phthalimido$\beta$-D-glucopyranosyl-(1 $\rightarrow 3)$-4-O-acetyl-2,6-di-O-benzo$y l$ - $\beta$-D-galactopyranosyl- $(1 \rightarrow 4)$ - 3-O-acetyl- 6 -O - tertbutyl-dimethylsilyl-2-deoxy-2-phthalimido-1-thio- $\beta$-Dglucopyranoside (14). - To a solution of compound 13 (560 $\mathrm{mg}, 0.427 \mathrm{mmol})$ in pyridine $(2 \mathrm{~mL})$ was added $\mathrm{Ac}_{2} \mathrm{O}(1 \mathrm{~mL})$. The mixture was stirred at $40^{\circ} \mathrm{C}$ for about $20 \mathrm{~h}$, then co-evaporated with toluene under diminished pressure to remove pyridine. The residue was purified by silica-gel column chromatography $(2: 1$ petroleum ether-EtOAc) to give syrupy $14(550 \mathrm{mg}$, 95.2\% $:[\alpha]_{\mathrm{D}}+41^{\circ}\left(c\right.$ 1.3, $\left.\mathrm{CHCl}_{3}\right) ;{ }^{1} \mathrm{H}$ NMR: -0.06 , $0.13\left(2 \mathrm{~s}, 2 \times 3 \mathrm{H}, 2 \mathrm{SiCH}_{3}\right), 0.81\left(\mathrm{~s}, 9 \mathrm{H}, \mathrm{C}\left(\mathrm{CH}_{3}\right)_{3}\right)$, $1.10,1.11\left(2 \mathrm{~d}, 6 \mathrm{H}, J 6.1,6.5 \mathrm{~Hz}, \mathrm{CH}\left(\mathrm{CH}_{3}\right)_{2}\right), 1.76$, $1.88,2.00,2.10,2.18\left(5 \mathrm{~s}, 5 \times 3 \mathrm{H}, 5 \mathrm{CH}_{3} \mathrm{CO}\right), 2.97(\mathrm{~m}$, $\left.1 \mathrm{H}, \mathrm{CH}\left(\mathrm{CH}_{3}\right)_{2}\right), 3.20\left(\mathrm{~m}, 1 \mathrm{H}, \mathrm{H}-5^{\mathrm{I}}\right), 3.47-3.55(\mathrm{~m}, 2$ $\left.\mathrm{H}, \mathrm{H}-6 \mathrm{a}^{\mathrm{I}}, \mathrm{H}-6 \mathrm{~b}^{\mathrm{I}}\right), 3.79-3.90$ (m, $4 \mathrm{H}, \mathrm{H}-4^{\mathrm{I}}, \mathrm{H}-3^{\mathrm{II}}, \mathrm{H}-5^{\mathrm{II}}$, $\left.\mathrm{H}-5^{\text {III }}\right), 4.08-4.22\left(\mathrm{~m}, 4 \mathrm{H}, \mathrm{H}-2^{\mathrm{I}}, \mathrm{H}-2^{\mathrm{III}}, \mathrm{H}-6 \mathrm{a}^{\mathrm{III}}, \mathrm{H}-\right.$ $\left.6 \mathrm{~b}^{\mathrm{III}}\right), 4.30\left(\mathrm{dd}, 1 \mathrm{H}, J_{6 \mathrm{a}, 6 \mathrm{~b}} 11.4, J_{6 \mathrm{a}, 5} 2.1 \mathrm{~Hz}, \mathrm{H}-6 \mathrm{a}^{\mathrm{II}}\right)$, $4.55\left(\mathrm{t}, 1 \mathrm{H}, J_{6 \mathrm{~b}, 5} 4.5 \mathrm{~Hz}, \mathrm{H}-6 \mathrm{~b}^{\mathrm{II}}\right), 4.74\left(\mathrm{~d}, 1 \mathrm{H}, J_{1,2} 8.1\right.$ $\left.\mathrm{Hz}, \mathrm{H}-1^{\mathrm{II}}\right), 5.13\left(\mathrm{t}, 1 \mathrm{H}, J_{4,3} 9.4, J_{4,5} 9.8 \mathrm{~Hz}, \mathrm{H}-4^{\mathrm{III}}\right), 5.19$ $\left(\mathrm{t}, 1 \mathrm{H}, J_{2,3} 9.8, J_{2,1} 8.1 \mathrm{~Hz}, \mathrm{H}-2^{\mathrm{II}}\right), 5.34\left(\mathrm{~d}, 1 \mathrm{H}, J_{1,2}\right.$ $\left.10.6 \mathrm{~Hz}, \mathrm{H}-1^{\mathrm{I}}\right), 5.44\left(\mathrm{~d}, 1 \mathrm{H}, J_{1,2} 8.3 \mathrm{~Hz}, \mathrm{H}-1^{\mathrm{III}}\right), 5.55$ (d, $\left.1 \mathrm{H}, J_{4,3} 3.6 \mathrm{~Hz}, \mathrm{H}-4^{\mathrm{II}}\right), 5.64\left(\mathrm{t}, 2 \mathrm{H}, J_{3,2} 10.5, J_{3,4} 9.4\right.$ $\left.\mathrm{Hz}, \mathrm{H}-3^{\mathrm{I}}, \mathrm{H}-3^{\mathrm{III}}\right), 7.11-8.15(\mathrm{~m}, 18 \mathrm{H}, \mathrm{Ph}) ;{ }^{13} \mathrm{C}$ NMR $\left(100 \mathrm{MHz}, \mathrm{CDCl}_{3}\right):-5.47,0.95\left(2 \mathrm{C}, \mathrm{SiCH}_{3}\right), 20.25$, 20.44, 20.53, 20.65, 20.68 (5 C, $\left.\mathrm{CH}_{3} \mathrm{CO}\right), 23.74,24.14$ (2 $\left.\mathrm{C}, \mathrm{CH}\left(\mathrm{CH}_{3}\right)_{2}\right), 25.71\left(4 \mathrm{C}, \mathrm{C}\left(\mathrm{CH}_{3}\right)_{3}\right), 34.81\left(\mathrm{CH}\left(\mathrm{CH}_{3}\right)_{2}\right)$, $54.27\left(\mathrm{C}-2^{\mathrm{III}}\right), 54.43\left(\mathrm{C}-2^{\mathrm{I}}\right), 60.88\left(\mathrm{C}-6^{\mathrm{I}}\right), 61.43\left(\mathrm{C}-6^{\mathrm{III}}\right)$, $62.45\left(\mathrm{C}-6^{\mathrm{II}}\right), 68.68\left(\mathrm{C}-4^{\mathrm{III}}\right), 69.24\left(\mathrm{C}-4^{\mathrm{II}}\right), 70.15\left(\mathrm{C}-3^{\mathrm{III}}\right)$, $71.01\left(\mathrm{C}-2^{\mathrm{II}}\right), 71.34\left(\mathrm{C}-3^{\mathrm{I}}\right), 71.36\left(\mathrm{C}-5^{\mathrm{III}}\right), 71.77\left(\mathrm{C}-5^{\mathrm{II}}\right)$, $74.57\left(\mathrm{C}-5^{\mathrm{I}}\right), 77.76\left(\mathrm{C}-3^{\mathrm{II}}\right), 78.83\left(\mathrm{C}-4^{\mathrm{I}}\right), 79.99\left(\mathrm{C}-1^{\mathrm{I}}\right)$, $98.39\left(\mathrm{C}-1^{\mathrm{III}}\right), 100.21\left(\mathrm{C}-1^{\mathrm{II}}\right), 163.83,166.16(2 \mathrm{C}$, PhCO), 166.45, 167.26, 167.61 (4 C, CO of Phth, some overlapped), 169.29, 169.91, 170.00, 170.65 (5 C, $\mathrm{CH}_{3} \mathrm{CO}$, some overlapped); MALDITOF-MS Calcd for $\mathrm{C}_{67} \mathrm{H}_{76} \mathrm{~N}_{2} \mathrm{O}_{24} \mathrm{SSi}: 1352$ [M]. Found: $1375.6[\mathrm{M}+\mathrm{Na}]^{+}$, $1391.6[\mathrm{M}+\mathrm{K}]^{+}$.

Methyl 3,4,6-tri-O-acetyl-2-deoxy-2-phthalimido- $\beta$-D - glucopyranosyl-(1 $\rightarrow 3)$-4-O-acetyl-2,6-di-O-benzoyl$\beta$-D-galactopyranosyl- $(1 \rightarrow 4)$-3-O-acetyl-6-O-tert-butyldimethylsilyl-2-deoxy-2-phthalimido- $\beta$-D-glucopyranosyl- $(1 \rightarrow 3)$ - 2,6-di-O-benzoyl- $\beta$ - D-galactopyranosyl$(1 \rightarrow 4)-2,3,4-t r i-O-b e n z y l-\alpha$-D-glucopyranoside (15).To a solution of compound $\mathbf{1 4}(530 \mathrm{mg}, 0.392 \mathrm{mmol})$ and $5(327 \mathrm{mg}, 0.392 \mathrm{mmol})$ in anhyd $\mathrm{CH}_{2} \mathrm{Cl}_{2}(4 \mathrm{~mL})$ was added NIS (220 mg, $0.98 \mathrm{mmol})$ and $\mathrm{Me}_{3} \mathrm{SiOTf}(21$ $\mu \mathrm{L}, 0.12 \mathrm{mmol}$ ) under an $\mathrm{N}_{2}$ atmosphere at $-15^{\circ} \mathrm{C}$. The mixture was stirred under these conditions for $1 \mathrm{~h}$. TLC (1:1 petroleum ether-EtOAc) indicated the completion of the reaction. The mixture was neutralized with $\mathrm{Et}_{3} \mathrm{~N}$ and concentrated. Purification of the residue on a silica gel column with $1: 1.3$ petroleum etherEtOAc as eluent gave $15(542 \mathrm{mg}, 65.5 \%)$ as a syrup: $[\alpha]_{\mathrm{D}}+34^{\circ}\left(c 1, \mathrm{CHCl}_{3}\right) ;{ }^{1} \mathrm{H} \mathrm{NMR}:-0.10,-0.03(2 \mathrm{~s}$, $\left.2 \times 3 \mathrm{H}, 2 \mathrm{SiCH}_{3}\right), 0.80\left(\mathrm{~s}, 9 \mathrm{H}, \mathrm{C}\left(\mathrm{CH}_{3}\right)_{3}\right), 1.76,1.79$, $1.99,2.10,2.16\left(5 \mathrm{~s}, 5 \times 3 \mathrm{H}, 5 \mathrm{CH}_{3} \mathrm{CO}\right), 3.09-3.19(\mathrm{~m}$, $\left.4 \mathrm{H}, \mathrm{H}-6 \mathrm{a}^{\mathrm{I}}, \mathrm{OCH}_{3}\right), 3.19-3.27\left(\mathrm{~m}, 2 \mathrm{H}, \mathrm{H}-5^{\mathrm{I}}, \mathrm{H}-5^{\mathrm{III}}\right)$, $3.28-3.39$ (m, 3 H, H-2, H-3 $\left.{ }^{\mathrm{II}}, \mathrm{H}-6 \mathrm{~b}^{\mathrm{I}}\right), 3.42(\mathrm{dd}, 1 \mathrm{H}$, $\left.J_{6 \mathrm{a}, 6 \mathrm{~b}} 11.4 \mathrm{~Hz}, \mathrm{H}-6 \mathrm{a}^{\mathrm{III}}\right), 3.48-3.57\left(\mathrm{~m}, 2 \mathrm{H}, \mathrm{H}-5^{\mathrm{II}}\right.$, H-6b $\left.{ }^{\text {III }}\right), 3.67-3.80\left(\mathrm{~m}, 6 \mathrm{H}, \mathrm{H}-3^{\mathrm{I}}, \mathrm{H}-4^{\mathrm{I}}, \mathrm{H}-4^{\mathrm{III}}, \mathrm{H}-3^{\mathrm{IV}}\right.$, $\left.\mathrm{H}-5^{\mathrm{IV}}, \mathrm{H}-5^{\mathrm{V}}\right), 3.94\left(\mathrm{~d}, 1 \mathrm{H}, J_{4,3} 4.6 \mathrm{~Hz}, \mathrm{H}-4^{\mathrm{II}}\right), 4.01-$ $4.26\left(\mathrm{~m}, 6 \mathrm{H}, \mathrm{H}-2^{\mathrm{III}}, \mathrm{H}-2^{\mathrm{V}}, \mathrm{H}-6 \mathrm{a}^{\mathrm{II}}, \mathrm{H}-6 \mathrm{a}^{\mathrm{IV}}, \mathrm{H}-6 \mathrm{a}^{\mathrm{v}}\right.$, one proton of $\left.\mathrm{PhCH}_{2}\right), 4.26-4.31\left(\mathrm{~m}, 2 \mathrm{H}, J_{1,2} 8.1 \mathrm{~Hz}\right.$, $\left.\mathrm{H}-1^{\mathrm{II}}, \mathrm{H}-6 \mathrm{~b}^{\mathrm{V}}\right), 4.44\left(\mathrm{~d}, 1 \mathrm{H}, J_{1,2} 3.6 \mathrm{~Hz}, \mathrm{H}-1^{\mathrm{I}}\right), 4.46-$ $4.61\left(\mathrm{~m}, 4 \mathrm{H}, \mathrm{H}-6 \mathrm{~b}^{\mathrm{II}}, \mathrm{H}-6 \mathrm{~b}^{\mathrm{IV}}\right.$, two protons of $\left.\mathrm{PhCH}_{2}\right)$, $4.64\left(\mathrm{~d}, 1 \mathrm{H}, J 10.8 \mathrm{~Hz}\right.$, one proton of $\left.\mathrm{PhCH}_{2}\right), 4.72(\mathrm{~d}$, $\left.1 \mathrm{H}, J_{1,2} 8.0 \mathrm{~Hz}, \mathrm{H}-1^{\mathrm{IV}}\right), 4.73,4.94(2 \mathrm{~d}, 2 \mathrm{H}, J 12.3$, $\left.10.8 \mathrm{~Hz}, \mathrm{PhCH}_{2}\right), 5.07-5.24\left(\mathrm{~m}, 3 \mathrm{H}, \mathrm{H}-2^{\mathrm{II}}, \mathrm{H}-2^{\mathrm{IV}}\right.$, H-4 $\left.{ }^{\mathrm{V}}\right), 5.31\left(\mathrm{~d}, 1 \mathrm{H}, J_{1,2} 8.4 \mathrm{~Hz}, \mathrm{H}-1^{\mathrm{III}}\right), 5.43(\mathrm{~d}, 1 \mathrm{H}$, $\left.J_{1,2} 8.3 \mathrm{~Hz}, \mathrm{H}-1^{\mathrm{v}}\right), 5.51\left(\mathrm{t}, 1 \mathrm{H}, J_{3,2} 10.7, J_{3,4} 9.1 \mathrm{~Hz}\right.$, $\left.\mathrm{H}-3^{\mathrm{III}}\right), 5.53\left(\mathrm{~d}, 1 \mathrm{H}, J_{4,3} 4.5 \mathrm{~Hz}, \mathrm{H}-4^{\mathrm{IV}}\right), 5.64(\mathrm{t}, 1 \mathrm{H}$, $\left.J_{3,2} 10.7, \mathrm{~J}_{3,4} 9.1 \mathrm{~Hz}, \mathrm{H}-3^{\mathrm{v}}\right), 7.09-8.10(\mathrm{~m}, 43 \mathrm{H}, \mathrm{Ph})$; ${ }^{13} \mathrm{C}$ NMR $\left(100 \mathrm{MHz}, \mathrm{CDCl}_{3}\right):-5.45,-5.07(2 \mathrm{C}$, $\left.\mathrm{SiCH}_{3}\right), 20.27,20.55,20.67\left(5 \mathrm{C}, \mathrm{CH}_{3} \mathrm{CO}\right.$, some overlapped), $25.63\left(4 \mathrm{C}, \mathrm{C}\left(\mathrm{CH}_{3}\right)_{3}\right), 54.43\left(\mathrm{C}-2^{\mathrm{V}}\right), 54.70$ 
$\left(\mathrm{C}-2^{\mathrm{III}}\right), 55.12\left(\mathrm{OCH}_{3}\right), 60.45\left(\mathrm{C}-6^{\mathrm{III}}\right), 60.47\left(\mathrm{C}-6^{\mathrm{V}}\right)$, $61.41\left(\mathrm{C}-6^{\mathrm{IV}}\right), 63.19\left(\mathrm{C}-6^{\mathrm{II}}\right), 67.38\left(\mathrm{C}-6^{\mathrm{I}}\right), 67.67\left(\mathrm{C}-4^{\mathrm{II}}\right)$, $68.62\left(\mathrm{C}-4^{\mathrm{V}}\right), 69.17\left(\mathrm{C}-5^{\mathrm{III}}\right), 69.29\left(\mathrm{C}-4^{\mathrm{IV}}\right), 69.99\left(\mathrm{C}-3^{\mathrm{III}}\right)$, $70.14\left(\mathrm{C}-3^{\mathrm{V}}\right), 70.85\left(\mathrm{C}-2^{\mathrm{II}}\right), 70.98\left(\mathrm{C}-2^{\mathrm{IV}}\right), 71.45\left(\mathrm{C}-5^{\mathrm{II}}\right)$, $71.55\left(\mathrm{C}-5^{\mathrm{V}}\right), 71.80\left(\mathrm{C}-5^{\mathrm{IV}}\right), 73.29\left(\mathrm{PhCH}_{2}\right), 73.42$ $\left(\mathrm{PhCH}_{2}\right), 74.00\left(\mathrm{C}-4^{\mathrm{III}}\right), 75.01\left(\mathrm{C}-5^{5}\right), 75.38\left(\mathrm{PhCH}_{2}\right)$, $76.04\left(\mathrm{C}-3^{\mathrm{I}}\right), 77.76\left(\mathrm{C}-3^{\mathrm{IV}}\right), 78.65\left(\mathrm{C}-2^{\mathrm{I}}\right), 79.76\left(\mathrm{C}-4^{\mathrm{I}}\right)$, $81.25\left(\mathrm{C}-3^{\mathrm{II}}\right), 98.37\left(2 \mathrm{C}, \mathrm{C}-1^{\mathrm{I}}, \mathrm{C}-1^{\mathrm{V}}\right), 98.57\left(\mathrm{C}-1^{\mathrm{III}}\right)$, $99.81\left(\mathrm{C}-1^{\mathrm{II}}\right), 100.11\left(\mathrm{C}-1^{\mathrm{IV}}\right), 163.80,164.09,166.13$, 166.17 (4 C, $\mathrm{PhCH}_{2}$ ), 166.60, 167.68, 167.72 (4 C, CO of Phth, some overlapped), 169.29, 169.91, 170.01, 170.65 (5 C, $\mathrm{CH}_{3} \mathrm{CO}$, some overlapped); MALDITOFMS Calcd for $\mathrm{C}_{112} \mathrm{H}_{118} \mathrm{~N}_{2} \mathrm{O}_{37} \mathrm{Si}$ : 2110 [M]. Found: $[\mathrm{M}+\mathrm{Na}]^{+}$2133.98; $[\mathrm{M}+\mathrm{K}]^{+}$2148.96. Anal. Calcd for $\mathrm{C}_{112} \mathrm{H}_{118} \mathrm{~N}_{2} \mathrm{O}_{37} \mathrm{Si}$ : C, 63.70; H, 5.63. Found: C, 64.02; $\mathrm{H}, 5.57$.

\section{Acknowledgements}

This work was supported by NNSF of China (Projects 29972053 and 39970179) and RCEES of CAS.

\section{References}

[1] Strecker, G.; Fievre, S.; Wieruszeski, J. M.; Michalski, J. C.; Montreuil, J. Carbohydr. Res. 1992, 226, 1-14 and the references cited therein.

[2] (a) Feizi, T. Nature 1985, 314, 53-57;

(b) Wieruszeski, J. M.; Chekkor, A.; Bouquelet, S.; Montreuil, J.; Strecker, G.; Peter-Katalinic, J.; Egge, H. Carbohydr. Res. 1985, 137, 127-138;

(c) Dua, V. K.; Goso, K.; Dube, V. E.; Bush, C. A. J. Chromatogr. 1985, 328, 259-269.

[3] Yamashita, K.; Mizuochi, T.; Kobata, A. Methods Enzymol. 1982, 83, 105-126.

[4] Saksena, R.; Deepak, D.; Khare, A.; Sahai, R.; Tripathi, L. M.; Srivastava, V. M. L. Biochem. Biophys. Acta 1999, 1428, 433-445.

[5] Catelani, G.; Colonna, F.; Marra, A. Carbohydr. Res. 1988, 182, 297-300.

[6] Garegg, P. J.; Hultberg, J.; Wallin, S. Carbohydr. Res. 1982, 108, 97-102.

[7] Du, Y.; Zhang, M.; Yang, F.; Gu, G. J. Chem. Soc., Perkin Trans. 1 2001, 3122-3127.

[8] Aguilera, B.; Fernandez-Mayoralas, A. J. Org. Chem. 1998, 63, 2719-2723.

[9] Du, Y.; Yang, F. Chinese Patent Appl. 01136484.x, 2001.

[10] Sato, S.; Ito, Y.; Nukada, T.; Nakahara, Y.; Ogawa, T. Carbohydr. Res. 1987, 167, 197-210. 